\section{Analysis of extracellular superoxide dismutase and Akt in ascending aortic aneurysm with tricuspid or bicuspid aortic valve}

\author{
A. Arcucci, ${ }^{1}$ M.R. Ruocco, ${ }^{2}$ F. Albano, ${ }^{1,2}$ \\ G. Granato, ${ }^{1,2}$ V. Romano, ${ }^{1}$ G. Corso, ${ }^{3}$ \\ C. Bancone, ${ }^{4}$ E. De Vendittis, ${ }^{2}$ \\ A. Della Corte, ${ }^{4}$ S. Montagnani ${ }^{1}$
}

'Department of Public Health, University of Naples Federico II

2Department of Molecular Medicine and Medical Biotechnologies, University of Naples Federico II

${ }^{3}$ Department of Clinical and Experimental Medicine, University of Foggia

${ }^{4}$ Department of Cardiothoracic Sciences, Second University of Naples, Italy

\section{Abstract}

Ascending aortic aneurysm (AsAA) is a consequence of medial degeneration (MD), deriving from apoptotic loss of smooth muscle cells (SMC) and fragmentation of elastin and collagen fibers. Alterations of extracellular matrix structure and protein composition, typical of medial degeneration, can modulate intracellular pathways. In this study we examined the relevance of extracellular superoxide dismutase (SOD3) and Akt in AsAA pathogenesis, evaluating their tissue distribution and protein levels in ascending aortic tissues from controls $(n=6)$, patients affected by AsAA associated to tricuspid aortic valve (TAV, $n=9$ ) or bicuspid aortic valve (BAV, $n=9$ ). The results showed a significant reduction of SOD3, phospho-Akt and Akt protein levels in AsAA tissues from patients with BAV, compared to controls, whereas the differences observed between controls and patients with TAV were not significant. The decreased levels of SOD3 and Akt in BAV aortic tissues are associated with decreased Erk1/Erk2 phosphorylation and MMP-9 levels increase. The authors suggest a role of decreased SOD3 protein levels in the progression of AsAA with BAV and a link between ECM modifications of aortic media layer and impaired Erk1/Erk2 and Akt signaling in the late stages of the aortopathy associated with BAV.

\section{Introduction}

Cardiovascular pathologies are the main causes of morbidity and mortality in western society. In particular, the incidence of supradiaphragmatic aorta aneurysms ranges from 5 to 10 cases per 100,000 individuals per year, with a peak incidence from the sixth to the seventh decade of life. ${ }^{1}$ Ascending aortic aneurysms (AsAAs), about $40 \%$ of aneurysms diagnosed, are not generally associated to atherosclerosis and most of them have an unknown aetiology. ${ }^{2,3}$ AsAAs are divided into three different forms: syndromic, familial non-syndromic, and degenerative. ${ }^{4}$ Bicuspid aortic valve (BAV) is a cardiac congenital abnormality, occurring in up to $2 \%$ of all the live births, associated to increased risk of both aortic valvulopathy and aortopathy in the adult life. ${ }^{3,4}$ However, all the different forms of ascending aortic aneurysm (AsAA) are the result of medial degeneration (MD), that is a pathological remodelling of the media layer. ${ }^{4}$ In fact, MD is characterized by a thinning of the tunica media due to the apoptotic loss of smooth muscle cells (SMC), fragmentation of elastin and collagen fibers and increased accumulation of mucoid material. ${ }^{4}$ Hence, both resident cells death and extracellular matrix (ECM) derangement are hallmarks of the aortopathies underlying aneurysm development and progression., Furthermore, the dysregulated vascular remodelling, specific of aneurysm development, is associated to modification of intracellular signaling in different cell types. ${ }^{2,5}$

The extracellular superoxide dismutase (SOD3) belongs to a family of metalloenzymes with superoxide dismutase activity. ${ }^{6-8}$ This protein is a copper and zinc enzyme, that removes superoxide anions from the extracellular microenvironment, via the dismutation of two moieties of this reactive oxygen species (ROS) to hydrogen peroxide and molecular oxygen., ${ }^{7,8}$ The SOD3 enzyme mantains the ECM homeostasis and, in physiological conditions, it is present to a great extent in aorta media layer. ${ }^{7-10}$ Moreover, SOD3 overexpression mediates anti-apoptotic signaling by inducing the activation of Akt and Ek1/2 pathways. ${ }^{11}$ Indeed, ECM protein composition and the signaling from ECM to cells, also referred as outside-in signalling, modulate intracellular pathways involved in a number of cellular functions. ${ }^{2,5}$ Akt affects cellular survival and proliferation, and, in aortic SMC, it regulates the expression of matrix metalloprotease-9 (MMP-9), a key proteinase in ECM destruction associated to MD. ${ }^{12,13}$ On the other hand studies focused on genetically modified mouse strains demonstrated that Erk1/Erk2 activation, induced by TGF- $\beta$ contributes to aortic aneurysm. ${ }^{14}$

In this study we examined the relevance of SOD3 and Akt in the late stages of the aortopathy associated with tricuspid aortic valve (TAV) or bicuspid aortic valve (BAV). To this aim, we evaluated SOD3 and Akt tissue distribution
Correspondence: Prof. Stefania Montagnani, Department of Public Health, University of Naples Federico II, via S. Pansini 5, 80131 Naples, Italy.

Tel. +39.081.7463421.

E-mail:montagna@unina.it

Key words: Ascending aortic aneurysm, bicuspid aortic valve, medial degeneration, smooth muscle cells, extracellular superoxide dismutase, Akt, tricuspid aortic valve.

Contributions: FA, GG, VR, experiments preforming; AA, MRR, GC, CB, EDV, data acquisition and analysis; AA, SM, manuscript writing; AA, MRR, ADC, SM, experiments design, and manuscript supervision; AA, MRR, contributed equally to the manuscript. All authors red and approved the final manuscript version.

Conflict of interests: the authors declare no conflict of interests.

Funding: this work was supported by a Grant from the Italian Health Ministry (GR 2009-1580439) and by a Grant from the Italian Ministry of University and Research (MIUR-PRIN 2009, prot. 2009P2HZZ7).

Received for publication: 4 February 2014 Accepted for publication: 11 June 2014

This work is licensed under a Creative Commons Attribution NonCommercial 3.0 License (CC BYNC 3.0).

\section{(C) Copyright A. Arcucci et al., 2014}

Licensee PAGEPress, Italy

European Journal of Histochemistry 2014; 58:2383 doi:10.4081/ejh.2014.2383

and their protein levels in ascending aorta tissues from control subjects and patients affected by AsAA with either TAV or BAV. Moreover we analysed the pErk1/Erk2 and MMP-9 levels in control ascending aorta tissues and in aneurysmal ascending aortic tissues.

\section{Materials and Methods}

\section{Aortic tissues}

Pathological aortic wall specimens were obtained from the outer curve (convexity) of the ascending aorta in patients undergoing elective surgical repair for AsAA, associated to TAV ( $\mathrm{n}=9,2$ males, 7 females, mean age $69 \pm 12$, aortic diamater $5.5 \mathrm{~cm} \pm 0.7$ ), or $\mathrm{BAV}$ $(\mathrm{n}=9,7$ males, 2 females, mean age $59 \pm 16$, aortic diameter $4.9 \mathrm{~cm} \pm 0.3$ ). Valve morphology (TAV/BAV) was defined on the basis of concordant echocardiographic diagnosis, surgical inspection and pathologic examination. 
Patients were selected on the basis of clinical factors known to affect SOD3 expression and/or vascular localization: ${ }^{7,8}$ patients with diabetes, coronary artery disease, hypercolesterolaemia or preoperative heparin therapy were excluded. Results were compared with nonaneurysmal aortic samples collected from 6 control subjects ( 3 heart transplant donors and 3 patients undergoing coronary artery bypass surgery with normal ascending aorta and aortic valve). The investigation conforms to the principles outlined in the Declaration of Helsinki and informed consent was obtained from all patients. Local Independent Ethical Committee approval was obtained.

All the fresh tissue specimens were washed in PBS solution and were divided in two aliquots. One aliquot, used for proteins extraction, was stored at $4^{\circ} \mathrm{C}$ overnight in RNAlater (Ambion Inc., Austin, TX, USA) and, without supernatant, moved to $-80^{\circ} \mathrm{C}$ for long-term storage. Another aliquot was formalin fixed for immunohistochemical analysis.

\section{Chemicals and reagents}

A protease inhibitor cocktail was obtained from Roche Diagnostics Corporation, (Indianapolis, IN, USA). Rabbit monoclonal antibody against $G A P D H$ was purchased from Cell SignalTechnology Inc. (Danvers, MA, USA); mouse monoclonal antibody against human SOD3 was obtained from AbCam, (Cambridge, UK); rabbit polyclonal antibody against human p-Akt 1/2/3 (Ser473), rabbit polyclonal antibody against human Akt 1, goat polyclonal antibody against $\beta$-actin, mouse monoclonal MMP-9 antibody, mouse monoclonal pErk antibody, rabbit polyclonal Erk antibody and each secondary antibody conjugated to horseradish peroxidase were obtained from Santa Cruz Biotechnology, Inc. (Santa Cruz, CA, USA). All other chemicals were of analytical grade and were purchased from SigmaAldrich (Saint Louis, M0, USA).

\section{Immunohistochemistry}

Aortic tissue samples were fixed in $10 \%$ neutral buffered formalin and embedded in paraffin (Bio-Optica Milano SpA, Milan, Italy), then sliced into serial $4 \mu \mathrm{m}$-thick sections and placed on poly-L-lysine coated glass slides (Menzel-Glaser, Brunswick, Germany). Slides were deparaffinised, rehydrated and immersed in $10 \mathrm{mM}$ citric acid, $\mathrm{pH} 6$, in a microwave oven (VWR International PBI Srl, Milan, Italy) to exclude epitope masking owing to fixation. Sections were immunostained with primary antibodies against SOD3 (1:10), Akt1 (1:100) and phospho-Akt1/2/3 (1:50) and detected with an indirect immunoperoxidase technique employing UltraVision LP Detection System HRP Polymer \& DAB Plus Chromogen (Thermo Fisher Scientific Inc., Waltham, MA, USA), according to the manufacturer's protocol. As negative controls, sections were incubated with PBS solution (Bio-Optica Milano SpA). Nuclear staining was carried out using hematoxylin. Microscopic analysis was performed with a Leica DMLB microscope (Leica Microsystems, Wetzlar, Germany). Pictures were taken in bright field with a digital camera (Leica DC200; Leica Microsystems) connected to the microscope.

\section{Tissue protein extracts and Western blotting analysis}

Protein extracts were obtained from the whole aortic wall. Briefly, $100 \mathrm{mg}$ of aortic tissues were homogenised in $500 \mu \mathrm{L}$ of extraction buffer ( $50 \mathrm{mM}$ Tris $\mathrm{HCl}$, pH 7.4, $5 \mathrm{mM}$ EDTA, $250 \mathrm{mM} \mathrm{NaCl}, 0.1$ \%Triton, $10 \mathrm{mM}$ DTT, $0.2 \mathrm{mM}$ PMSF, supplemented with protease inhibitor cocktail) on ice and then samples were incubated for $30 \mathrm{~min}$ at $4^{\circ} \mathrm{C}$. The supernatants obtained after centrifugation at 14,000 $\mathrm{g}$ for $30 \mathrm{~min}$ at $4^{\circ} \mathrm{C}$ were collected and constituted the total protein extracts. Protein concentration was determined by the Bradford assay using bovine serum albumin as standard. ${ }^{15}$ Western blotting analysis was performed with $20 \mu \mathrm{g}$ of total protein extracts. Briefly, protein samples were dissolved in SDSreducing loading buffer, run on a SDS/PAGE and then transferred to Immobilon $\mathrm{P}$ membrane (Millipore Corporation, Billerica, MA, USA). The filter was incubated with the specific primary antibody at $4^{\circ} \mathrm{C}$ overnight and then with the horseradish peroxidase-linked secondary antibody at room temperature for $1 \mathrm{~h}$. In particular, for pAkt and pErk protein levels analysis, filters were incubated with anti-pAkt and anti-pErk primary antibodies and with the horseradish peroxidase-linked secondary antibodies. Membranes were then analyzed by an enhanced chemiluminescence reaction, using WesternBright ECL-HRP substrate (Advansta, Mello Park, CA, USA) according to the manufacturer's instructions; signals were visualized by autoradiography. Filters were then stripped, using AbCam stripping solution, pH 2 (200 mM Glycine, $3.5 \mathrm{mM} \mathrm{SDS}, 1 \%$ Tween-20) at $45^{\circ} \mathrm{C}$ for $30 \mathrm{~min}$ and washed 3 times with PBSTween $0.1 \%$. Blocking was performed again, before incubation with anti-Akt and anti-Erk primary antibodies. Membranes were then analyzed by an enhanced chemioluminescence reaction, as previously specified. Densitometric analysis of band intensity was performed using the Image J 1.48i version (Wayne Rasband National Institutes of Health, USA).

\section{Gelatin gel zymography}

MMP-9 activity was measured using gelatin gel zymography. Gelatinolytic activity in $30 \mu \mathrm{g}$ of total protein extracts was detected by SDSPAGE zymography. Samples were analysed under non reducing conditions without boiling, through a $10 \%$ SDS-polyacrylamide gel copolymerized in presence of gelatin $(2 \mathrm{mg} / \mathrm{mL})$ for gelatinolytic zymography. After the electrophoresis run, carried out at $35 \mathrm{~mA}$ for 150 $210 \mathrm{~min}$ at $4^{\circ} \mathrm{C}$, the gels were washed, renatured in a $2.5 \%$ Triton X-100 solution for $1 \mathrm{~h}$. The gels were then incubated in developing buffer containing $50 \mathrm{mM}$ Tris- $\mathrm{HCl}, \mathrm{pH}$ 7.5, 200 $\mathrm{mM} \mathrm{NaCl}, 5 \mathrm{mM} \mathrm{CaCl} 2$ and $5 \mu \mathrm{M} \mathrm{CaCl}_{2}$ at $37^{\circ} \mathrm{C}$ for $48 \mathrm{~h}$, which allows substrate degradation. Finally, the gels were fixed in 30\% methanol, $10 \%$ acetic acid for 30 min, stained with $0.1 \%$ Coomassie Brillant Blue R-250. Proteolytic bands were visualized by destaining with $50 \%$ methanol and 5\% acetic acid. The level of MMP-9 enzymatic activity was determined by quantifying clear band corresponding in size.

\section{Statistical analysis}

Data are reported as mean \pm standard deviation (SD). The statistical significance of differences between groups was evaluated using one-way ANOVA with Bonferroni corrections. A P-value of $<0.05$ was considered statistically significant.

\section{Results}

\section{Analysis of SOD3 and pErk1/Erk2 proteins}

We first analysed the SOD3 distribution and protein levels in tissues from patients with AsAA and control subjects. To this aim, the aortic specimens from control subjects $(n=6)$ and patients with AsAA associated to TAV $(n=9)$ or $\operatorname{BAV}(n=9)$ were assayed by immunohistochemistry and immunoblotting for the evaluation of SOD3 distribution and protein levels (Figures 1 and 2). Hence, immunohistochemical analysis was carried out on nonaneurysmal and pathological ascending aortic tissues (Figure 1). Immunostaining for SOD3 protein was observed both in ECM and inside the cells of tunica media from ascending aortic tissues of control subjects and TAV patients (Figure 1 A,B), whereas no SOD3 staining was evident in AsAA tissues from BAV patients (Figure 1C). Western blotting analysis of SOD3 in the protein extracts of the whole aortic wall showed an evident difference in SOD3 protein levels between nonaneurysmal and BAV pathological samples (Figure 2A). However, the densitometric analysis showed that SOD3 levels decreased also in TAV pathological samples (Figure 2B). In particular, SOD3 levels observed in BAV aortic tissues were about three times lower compared to nonaneurysmal tissues $(\mathrm{P}<0.05)$, whereas SOD3 levels in TAV samples were not significantly different compared to controls $(\mathrm{P}>0.05)$ (Figure $2 \mathrm{~B})$. 
It is known that the overexpression of SOD3, in skeletal muscle ischemia model, promotes cell survival, tissue recovery, and correlates with activation of Erk1/Erk2. ${ }^{11}$ Hence, to test if there is a correlation between SOD3 protein levels and Erk1/Erk2 activation in ascending aortic tissues, we evaluated, by Western blotting analysis, the levels of Erk1/Erk2 activation in normal and pathological aortic specimens. As shown in Figure 3, a pErk1/2 decrease was detected in samples from BAV patients compared to control subjects $(\mathrm{P}<0.01)$, whereas that observed in TAV samples was not significant $(\mathrm{P}>0.05)$.

\section{Analysis of Akt tissue distribution and protein levels}

The distribution of Akt in tissues from AsAA patients was examined by immunohistochemical analysis (Figure 4). In particular, a clear intracellular phospho-Akt (pAkt) staining was detected in the media layer of both controls (Figure 4A) and aneurismal TAV tissues (Figure 4B); conversely, intracellular pAkt immunostaining was not detectable around nuclear hematoxylin staining of aneurismal BAV tissues (Figure 4C). Concerning Akt, its intracellular staining was evident in controls (Figure 4D) and TAV specimens (Figure 4E), whereas it was hardly detectable in BAV tissues (Figure 4F).

The comparison of pAkt and Akt levels in protein extracts from nonaneurysmal ascending aortic wall and AsAA associated to TAV or $\mathrm{BAV}$ is shown in Figure 5. A clear reduction of pAkt level is evident in samples from BAV patients (Figure 5A). Indeed, the densitometric analysis (Figure 5B) showed that the level of pAkt and the ratio of pAkt/Akt were decreased in tissues from BAV patients ( $\mathrm{P}<0.001$ and $\mathrm{P}<0.01$, respectively) compared to tissues of control subjects, indicating a lesser activation of Akt in AsAA tissues from BAV patients. Moreover Western blotting and densitometric analysis showed also Akt level decrease in BAV tissues, compared to control tissues $(\mathrm{P}<0.05)$. When the aortic specimens from TAV patients were compared with those from control subjects, a smaller, not significant decrease of pAkt, Akt and pAkt/Akt ratio emerged (Figure 5B). Altogether, immunohistochemical and Western blotting results indicate that pAkt, Akt levels and pAkt/Akt ratio are significantly reduced in AsAAs with BAV and that the signalling pathway of Akt may be impaired in these tissues.

\section{Analysis of MMP-9 protein levels and enzymatic activity}

It is known that expression and activation of Akt downregulates MMP-9 protein levels in human aortic SMC. ${ }^{13}$ Hence, to verify if the reduction of Akt activation, observed in BAV patients, is associated to an increase of MMP9 levels, the aortic specimens from control subjects and patients with AsAA were assayed by immunoblotting and gelatin gel zymography for the evaluation of MMP-9 protein levels and enzymatic activity, respectively (Figures 6 and 7). Western blotting and its densitometric analysis demonstrated a clear increase of MMP-9 protein levels in AsAA tissues from BAV patients (Figure $6 \mathrm{~A}, \mathrm{~B}$ ). In particular, MMP-9
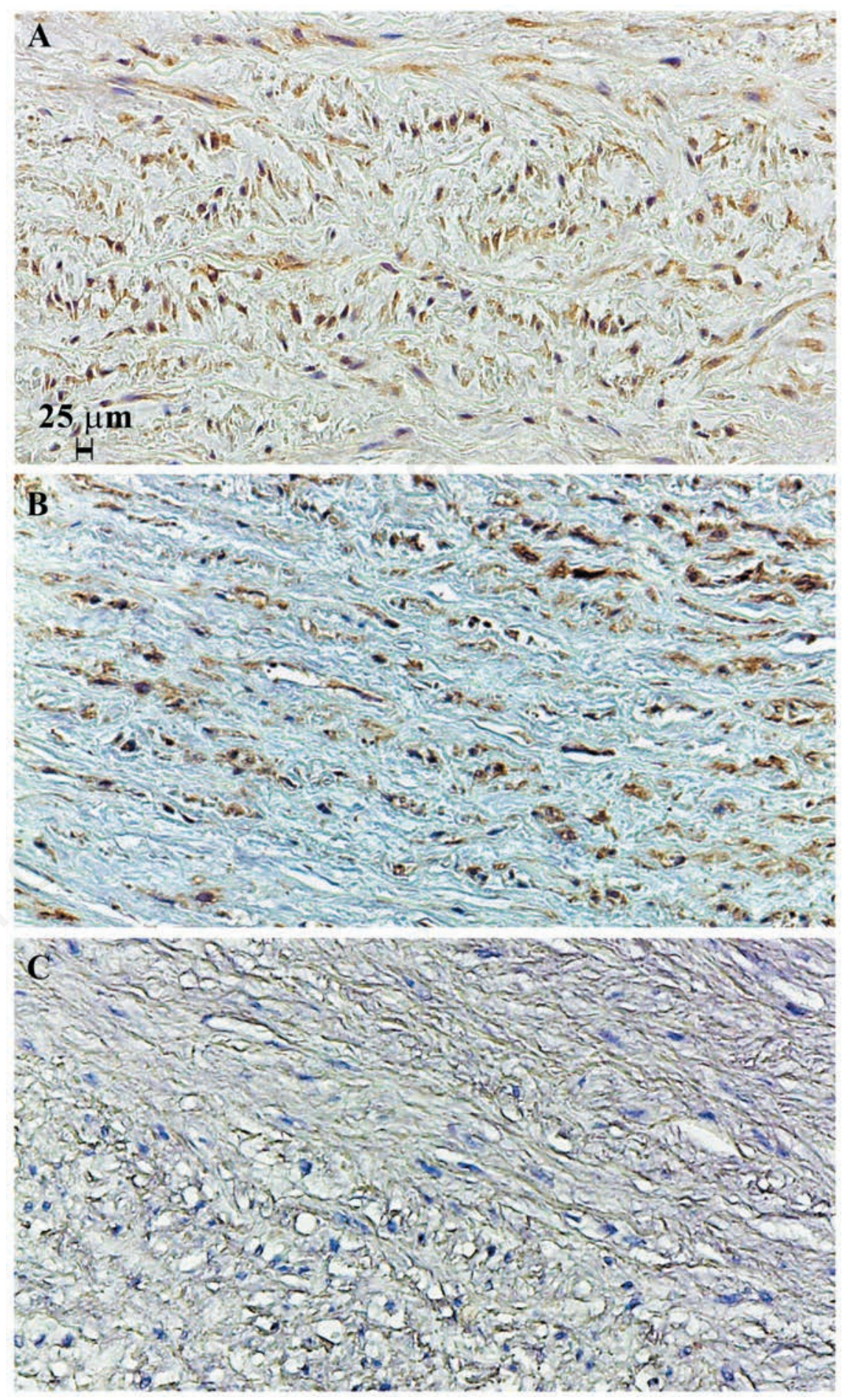

Figure 1. Immunohistochemical analysis of SOD3 protein. Immunostaining of SOD3 in nonaneurysmal ascending aortic media layer (A). Immunostaining of SOD3 in ascending aortic media layer from patients affected by AsAA associated to TAV (B) or BAV (C). Representative images are shown. 
protein levels were increased in tissues from BAV patients compared to tissues of control subjects and TAV patients $(\mathrm{P}<0.05)$ (Figure $6 \mathrm{~B})$. When the aortic specimens from TAV patients were compared with those from control subjects no difference emerged. Furtehermore, gelatin gel zymography (Figure $7 \mathrm{~A}, \mathrm{~B})$ demonstrated a great increase of MMP9 enzymatic acivity in tissues from BAV patients compared to tissues of control subjects and TAV patients $(\mathrm{P}<0.01)$.

\section{Discussion}

Maladaptive vascular remodelling within the media aortic layer leads to $\mathrm{MD}$, a process that at least in BAV patients affects particularly the convexity of the ascending aorta (greater curvature); as a consequence, the media layer structural integrity is lost and this causes the aneurysm formation, characterized, in patients with associated aortic valve disease, by expansion of the greater curvature predominantly. ${ }^{1,3,16}$ The aortic media layer provides the required vasal viscoelasticity through concentric bands of elastin filaments with associated collagen fibers and SMC; these structures are termed lamellar units. ${ }^{17}$ Thirty two lamellar units are present in the media of abdominal aorta; these structures do not contain blood vessels and represent an avascular zone, receiving oxygen and nutrients only by means of transintimal diffusion from the plasma. ${ }^{17} \mathrm{On}$ the contrary, the media layer of the ascending aorta is formed by nearly sixty lamellar units and contains, in its outer zone, vasa vasorum penetrating the media from the adventitia, thus creating a vascular zone. ${ }^{17}$ In particular, the vascularization of ascending aorta outer media is responsible for the high levels of oxygen and derived ROS. Therefore, the ascending aortic media layer requires efficient antioxidant enzyme systems to control both intracellular and ECM redox state.

We observed that SOD3 protein levels are strongly reduced in AsAAs associated to BAV patients with respect to nonaneurysmal ascending aortic tissues. Immunohistochemical analysis demonstrated the presence of SOD3 in both ECM and cells of tunica media from nonaneurysmal ascending aorta and TAV aortic tissues, but failed to detect any SOD3 immunostaning in BAV tissues. The absence of SOD3 immunostaining in the tunica media of BAV pathological ascending aorta tissues is explained by the significant and dramatic reduction of SOD3 protein levels showed by Western blotting analysis. Hence the strong reduction of SOD3 levels observed in BAV tissues, compared to nonaneurysmal tissues, could be a distinctive marker of the late stages
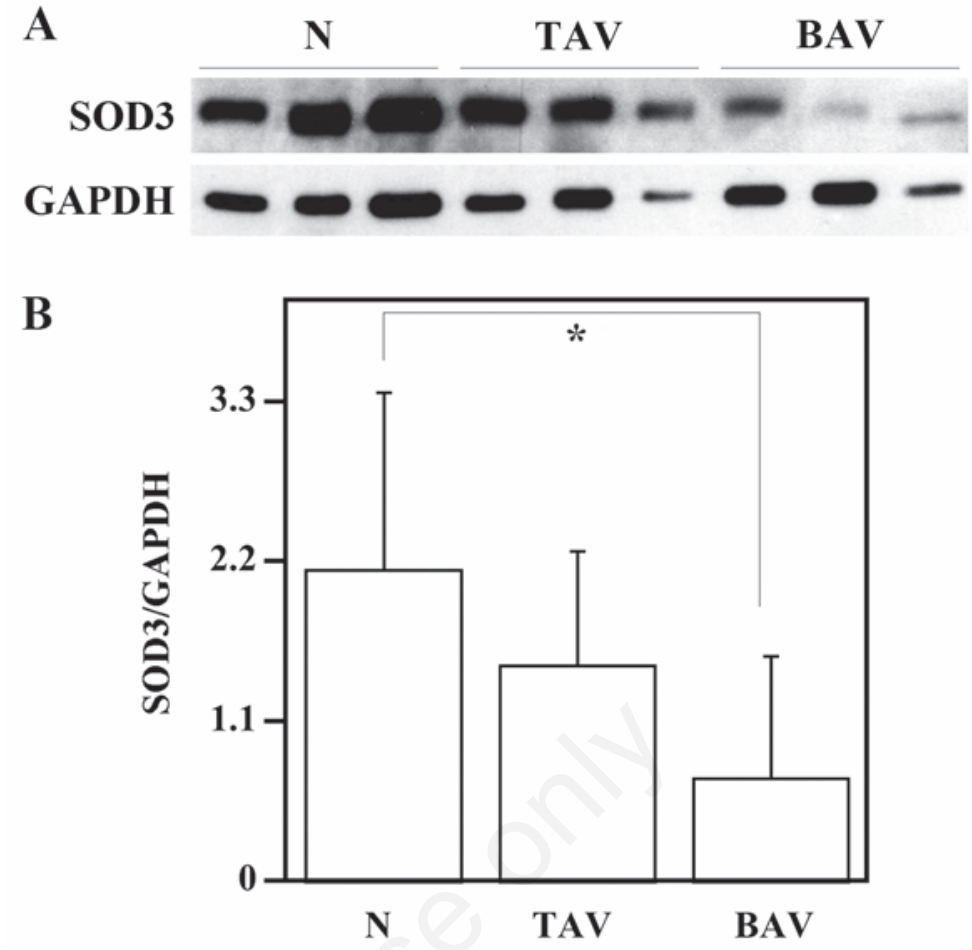

Figure 2. Analysis of SOD3 protein levels in the ascending aortic wall. Western blotting analysis, for SOD3 protein levels evaluation, was performed on ascending aorta protein extracts from six nonaneurysmal aortas $(\mathrm{N})$, nine patients with AsAA associated to TAV and nine patients with AsAA associated to $\mathrm{BAV}(\mathrm{A})$; GAPDH was used as loading control. Representative image is shown. Densitometric analysis of SOD3 protein levels (B). Data are reported as mean $\pm S D . * P<0.05$.

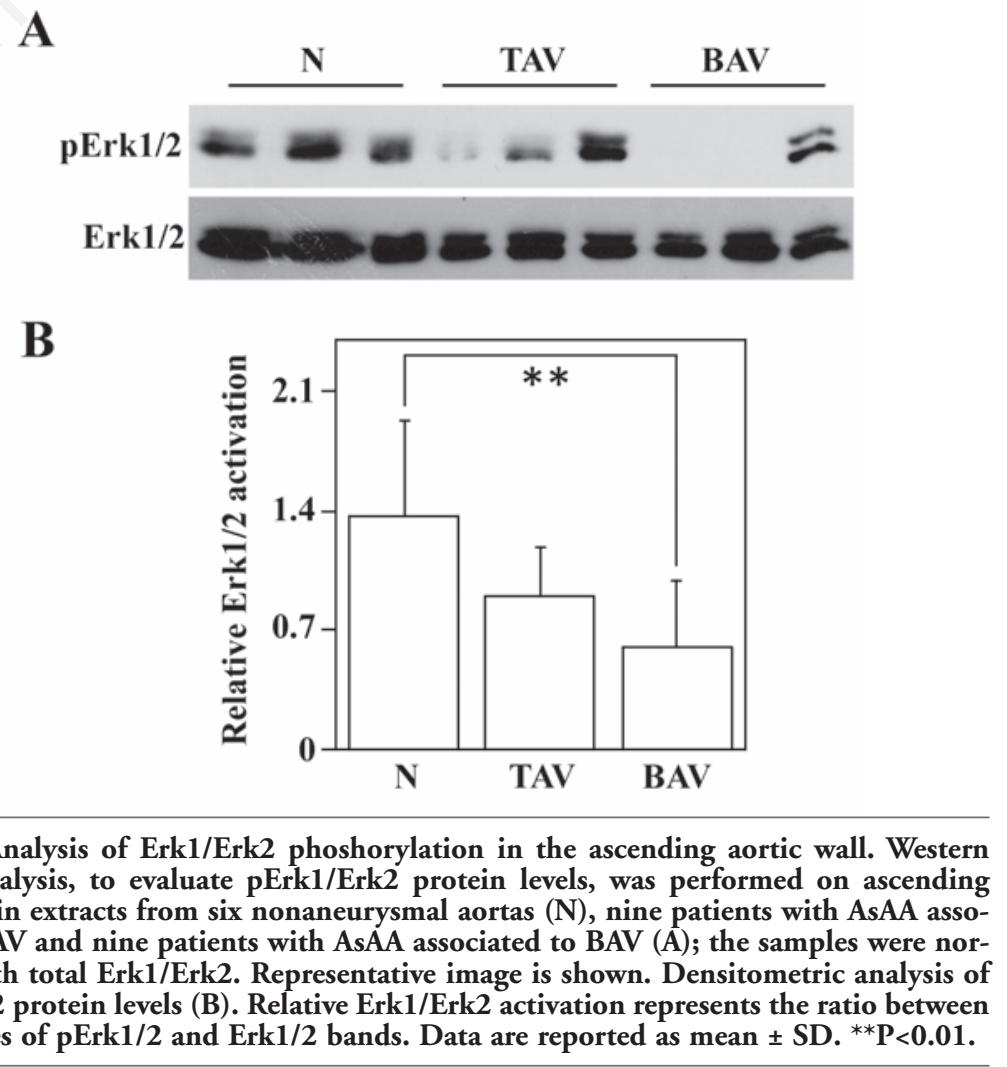


of the aortopathy associated with BAV. Furthermore, it is known that SOD3 binds to type I collagen and protects this structural protein from oxidative fragmentation; ${ }^{10}$ therefore, the reduction of SOD3 levels detected in BAV aortic specimens could be correlated also to the ECM structural modifications in the AsAA media layer. Besides the extracellular location of SOD3, our study suggested the presence of this enzyme also within the cells as evidenced by a previous study; ${ }^{9}$ however, the biological meaning of intracellular SOD3 localization is still unknown.

Previous publication has demonstrated that in patients affected by coronary artery disease SOD3 activity is reduced. ${ }^{18}$ Laatikainen et al. showed that SOD3 overexpression mediates anti-apoptotic signaling in skeletal muscle ischemia model and correlates with simultaneous activation of Erk1/2 and Akt. ${ }^{11}$ Furthermore, it has been shown that RasErk1/2 pathway, activated by the dismutase reaction end product, $\mathrm{H}_{2} \mathrm{O}_{2}$, is a major signal transduction cascade controlling SOD3 production and then Erk activation and SOD3 expression might be coordinately regulated through a positive feedback loop, most likely through $\mathrm{H}_{2} \mathrm{O}_{2} \cdot{ }^{19}$ In our experimental system decreased SOD3 protein levels detected in the aortic wall of AsAA from BAV patients is associated with decreased Erk1/Erk2 phosphorylation: hence, our data suggest that SOD3 production and Erk1/Erk2 activation could be regulated through a feedback loop. It is known that in Marfan syndrome mice noncanonical TGF $\beta$ dependent signaling, associated with Erk1/Erk2 activation, has a critical role in aneurysm pathogenesis and that MMP-2 activates latent TGF- $\beta$ and augments the noncanonical signaling cascade downstream, including Erk1/Erk2 activation. ${ }^{14,20} 0$ n the contrary our data indicate that Erk1/2 activation does not contribute to AsAA associated to BAV. However other studies will be required to clarify the biological role of feedback loop coupling SOD3 with Erk1/2 activation in physiopathology of ascending aorta.

It is known that the dysregulated signalling from ECM to the cells of aortic media layer could depend on molecular, architectural and redox alterations of $\mathrm{ECM} ;{ }^{2}$ here we show that activation of Akt is dramatically reduced in BAV pathological aortic tissues. The Akt kinase family comprises three highly homologous forms, controlling many cellular functions such as cell growth, survival, proliferation, glucose and lipid homeostasis. ${ }^{12}$ Akt1 is widely distributed in tissues and regulates cell growth and survival; in particular, in cardiovascular apparatus Aktl is the most important form affecting relevant biological functions. ${ }^{12,21,22}$ Furthermore, Akt pathway is activated by SOD3 overexpression and $\mathrm{H}_{2} \mathrm{O}_{2} \cdot{ }^{11,23}$ Hence, the strong
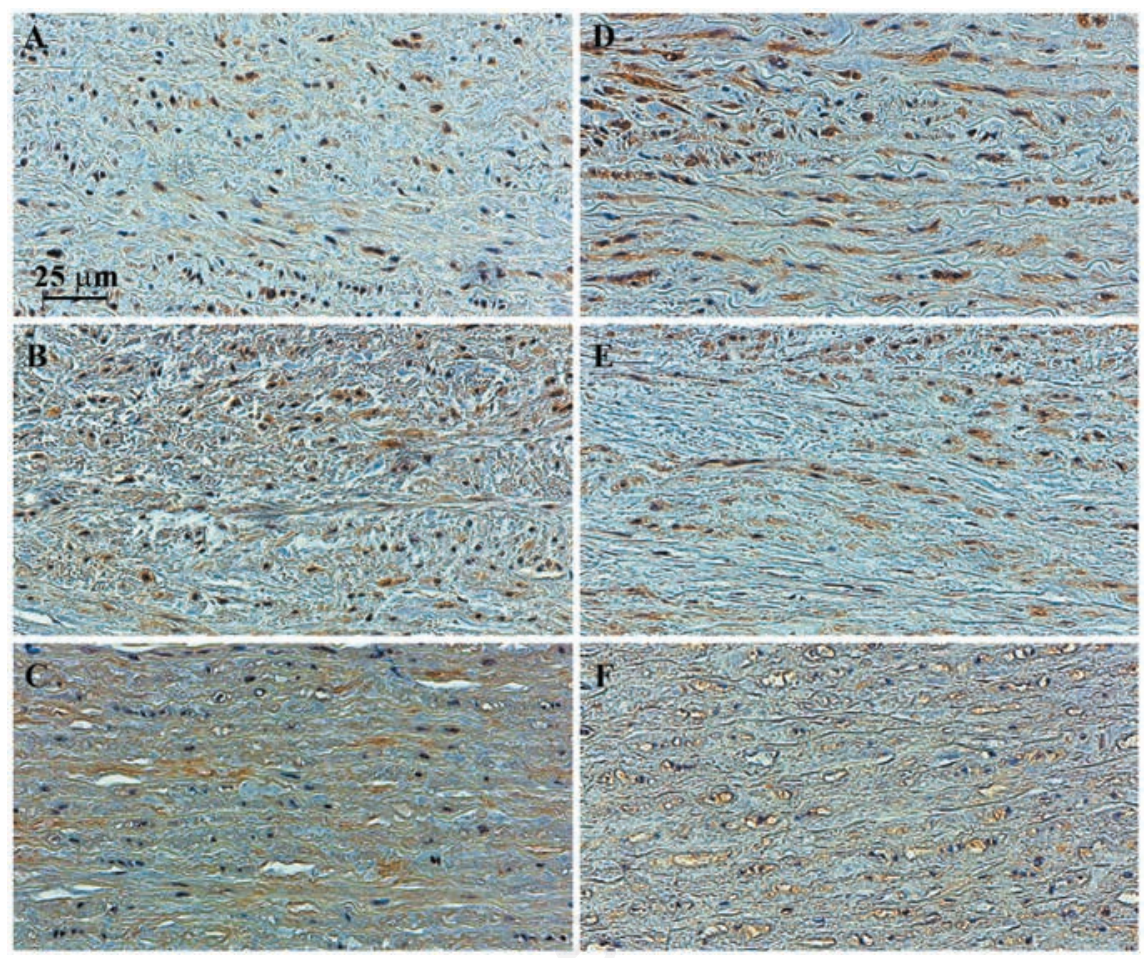

Figure 4. Immunostaining of pAkt and Akt intracellular proteins in control and pathological ascending aortic media layer. Immunohistochemical analysis of pAkt and Akt was performed in nonaneurysmal ascending aortic media layer $(A, D)$, in ascending aortic media layer from patients with AsAA associated to TAV (B, E) or BAV (C, F). Representative images are shown.

A

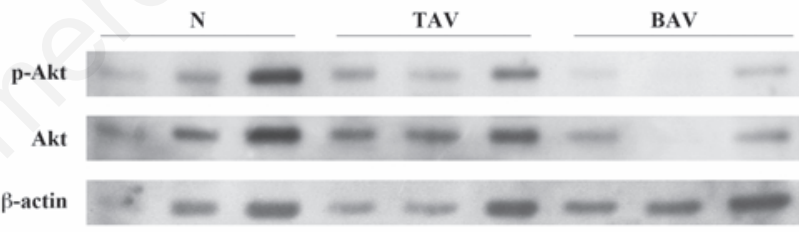

B
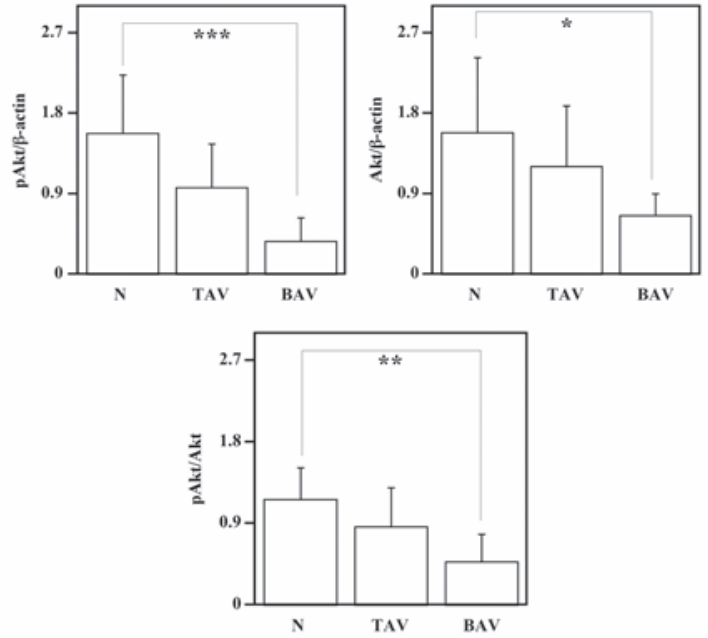

Figure 5. Analysis of pAkt and Akt levels in the ascending aortic wall. Comparison, by Western blotting analysis, of pAkt and Akt protein levels between ascending aortic tissues from six nonaneurysmal aortas $(\mathrm{N})$, nine patients with AsAA associated to TAV and nine patients with AsAA associated to BAV (A). $\beta$-actin was used as loading control. Representative image is shown. Densitometric analysis (B). Densities of pAkt and Akt bands were measured and normalized respect to the densities of $\beta$-actin bands. pAkt/Akt represents the ratio between the densities of pAkt and Akt bands. Data are reported as mean $\pm \mathrm{SD} .{ }^{*} \mathbf{P}<\mathbf{0 . 0 5},{ }^{* *} \mathbf{P}<\mathbf{0 . 0 1},{ }^{* * *} \mathrm{P}<\mathbf{0 . 0 0 1}$. 
reduction of SOD3 in BAV AsAA tissues, leading to a decreased production of the extracellular $\mathrm{H}_{2} \mathrm{O}_{2}$, could have an influence on both modifications in the ECM redox state and activation of Erk1/Erk2 and Akt.

In human aortic SMC, expression and activation of Akt reduces MMP-9 levels. ${ }^{13}$ In our experimental system, the reduction of Akt activation and protein levels, showed in BAV tissues, is associated with a significant increase of MMP-9 protein levels and enzymatic activity. MMPs are a family of proteases regulating the homeostasis of connective tissue. MMP-2 and MMP-9 are expressed in ascending aorta, and degrade type IV collagen and partially degrade elastin and fibrillar collagens. ${ }^{3}$ MMP-2 and MMP-9 are synthesized by vascular SMC, in response to hemodynamic changes and various disease states. ${ }^{3}$ MMPs activity may be inhibited by tissue inhibitors of metalloproteinase (TIMP); ${ }^{3}$ TIMP, of which TIMP-1 is the most common in the aorta, are synthesized by vascular SMC and fibroblasts. ${ }^{3}$ MMPs are important factors in the development of AsAA, and in particular Phillippi et al. showed increased MMP-9 expression in BAV-associated AsAA tissues compared to control tissues. ${ }^{24}$ Thus, in our experimental system, the reduction of Akt activation and protein levels, showed in BAV tissues, can be associated with MD of BAV aortic tissues and specifically with increased MMP-9 levels.

However it is noteworthy that shear stress, product of blood viscosity and velocity, has a role in pathogenesis of BAV aortic disease, is associated with cellular signalling cascades, resulting in increased expression of MMPs and growth factors that affect matrix degradation and SMC apoptosis; ${ }^{3}$ moreover shear stress changes the activation patterns of Akt and Erk. ${ }^{25}$ Therefore, it is possible that differences in hemodynamic and wall stress between nonaneurysmal ascending aorta, AsAA with TAV or BAV could further explain the detected alterations of SOD3, pErk1/Erk2, pAkt and MMP-9 levels.

In conclusion, although further studies will be required to elucidate the biological relevance of SOD3 and its interaction with Erk1/Erk2 and Akt pathways in AsAA with BAV, our study is the first to suggest a role of decreased SOD3 protein levels in the progression of AsAA with BAV and a link between ECM modifications of aortic media layer and impaired Erk1/Erk2 and Akt signaling in the late stages of the aortopathy associated with BAV. Moreover our data support the hypothesis that AsAA in TAV and BAV patients results from different signaling pathways, molecular and cellular mechanisms. ${ }^{26,27}$
A

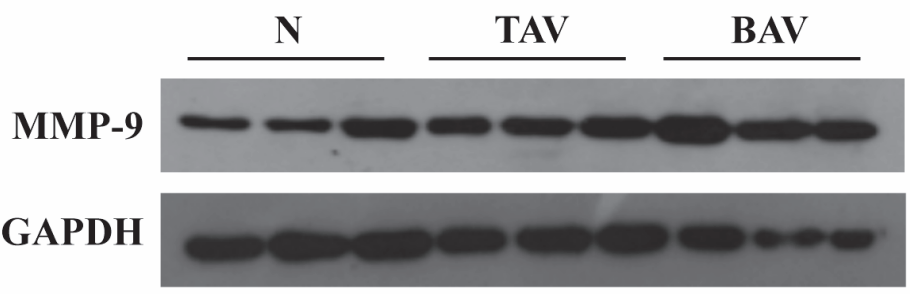

B

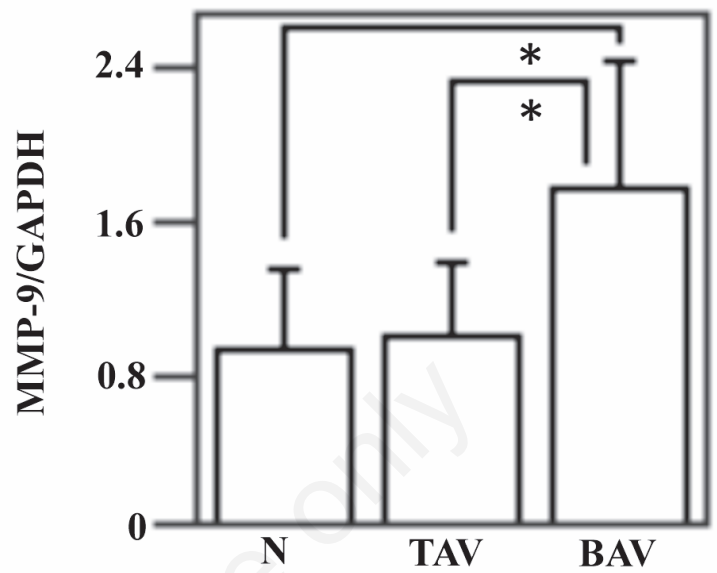

Figure 6. Analysis of MMP-9 protein levels in the ascending aortic wall. Western blotting analysis (A) of MMP-9 protein levels, was performed on ascending aorta protein extracts from six nonaneurysmal aortas $(\mathrm{N})$, nine patients with AsAA associated to TAV and nine patients with AsAA associated to BAV; GAPDH was used as loading control (A). Representative image is shown. Densitometric analysis of MMP-9 protein levels (B). Data are reported as mean $\pm \mathrm{SD}$. ${ }^{*} \mathrm{P}<0.05$.

A

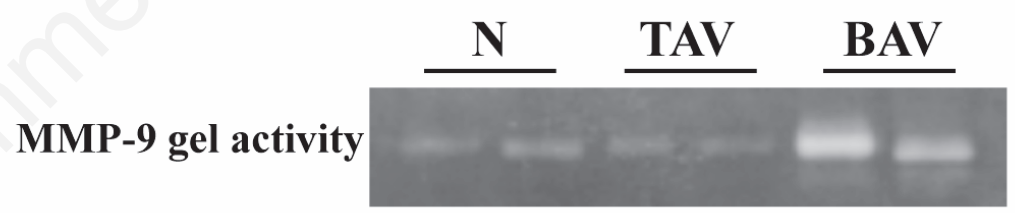

B

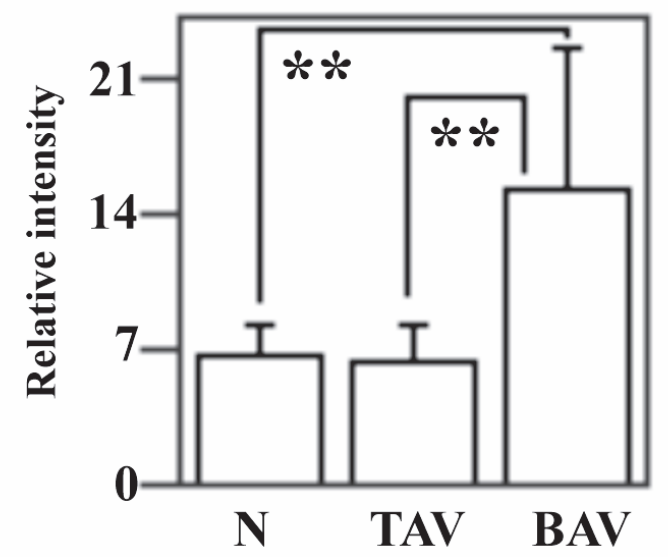

Figure 7. Analysis of MMP-9 enzymatic activity in the ascending aortic wall. Gelatin gel zymography was performed on ascending aorta protein extracts from six nonaneurysmal aortas (N), nine patients with AsAA associated to TAV and nine patients with AsAA associated to BAV (A). Representative image is shown. MMP-9 activity was determined by densitometric analysis of clear band corresponding in size (B). Data are reported as mean $\pm \mathrm{SD} .{ }^{* *} \mathrm{P}<\mathbf{0 . 0 1}$. 


\section{References}

1. Lavall D, Schäfers HJ, Böhm M, Laufs U. Aneurysms of the ascending aorta. Dtsch Arztebl Int 2012;109:227-33.

2. Jones JA, Spinale FG, Ikonomidis JS. Transforming growth factor-beta signaling in thoracic aortic aneurysm development: a paradox in pathogenesis. J Vasc Res 2009;46:119-37.

3. Tadros TM, Klein MD, Shapira OM. Ascending aortic dilatation associated with bicuspid aortic valve: pathophysiology, molecular biology, and clinical implications. Circulation 2009;119:880-90.

4. Blunder S, Messner B, Aschacher T, Zeller I, Turkcan A, Wiedemann D, et al. Characteristics of TAV- and BAV-associated thoracic aortic aneurysms-smooth muscle cell biology, expression profiling, and histological analyses. Atherosclerosis 2012;220:355-61.

5. Forte A, Della Corte A, De Feo M, Cerasuolo F, Cipollaro M. Role of myofibroblasts in vascular remodelling: focus on restenosis and aneurysm. Cardiovasc Res 2010;88:395-405.

6. Albano F, Arcucci A, Granato G, Romano S, Montagnani S, De Vendittis E, et al. Markers of mitochondrial dysfunction during the diclofenac-induced apoptosis in melanoma cell lines. Biochimie 2013; 95:934-45.

7. Fukai T, Ushio-Fukai M. Superoxide dismutases: role in redox signaling, vascular function, and diseases. Antioxid Redox Signal 2011;15:1583-606.

8. Nozik-Grayck E, Suliman HB, Piantadosi CA. Extracellular superoxide dismutase. Int J Biochem Cell Biol 2005;37:2466-71.

9. Arcucci A, Ruocco MR, Amatruda N, Riccio A, Tarantino G, Albano F, et al. Analysis of extracellular superoxide dismutase in fibroblasts from patients with systemic sclerosis. J Biol Regul Homeost Agents 2011;25:647-54.

10. Petersen SV, Oury TD, Ostergaard L, Valnickova Z, Wegrzyn J, Thogersen IB, et al. Extracellular superoxide dismutase (EC-SOD) binds to type I collagen and protects against oxidative fragmentation. $\mathrm{J}$ Biol Chem 2004:279:13705-10.

11. Laatikainen LE, Incoronato M, Castellone MD, Laurila JP, Santoro M, Laukkanen M0. SOD3 decreases ischemic injury derived apoptosis through phosphorylation of Erk1/2, Akt, and Fox03a. PLoS One 2011;6:e24456.

12. Hers I, Vincent EE, Tavarè JM. Akt signalling in health and disease. Cell Signal 2011;23:1515-27.

13. Shen YH, Zhang L, Ren P, Nguyen MT, Zou $\mathrm{S}$, Wu D, et al. AKT2 confers protection against aortic aneurysms and dissections. Circ Res 2013;112:618-32.

14. Holm TM, Habashi JP, Doyle JJ, Bedja D, Chen Y, van Erp C, et al. Noncanonical TGF- $\beta$ signaling contributes to aortic aneurysm progression in Marfan syndrome mice. Science 2011 332:358-61.

15. Bradford MM. A rapid and sensitive method for the quantitation of microgram quantities of protein utilizing the principle of protein-dye binding. Anal Biochem 1976;72:248-54.

16. Della Corte A, Quarto C, Bancone C, Castaldo C, Di Meglio F, Nurzynska D, et al. Spatiotemporal patterns of smooth muscle cell changes in ascending aortic dilatation with bicuspid and tricuspid aortic valve stenosis: focus on cell-matrix signaling. J Thorac Cardiovasc Surg 2008;135:8-18.

17. Ruddy JM, Jones JA, Spinale FG, Ikonomidis JS. Regional heterogeneity within the aorta: relevance to aneurysm disease. J Thorac Cardiovasc Surg 2008; 136:1123-30.

18. Landmesser U, Merten R, Spiekermann S, Buttner K, Drexler H, Hornig B. Vascular extracellular superoxide dismutase activity in patients with coronary artery disease: relation to endothelium-dependent vasodilation. Circulation 2000;101:2264-70.

19. Laurila JP, Castellone MD, Curcio A, Laatikainen LE, Haaparanta-Solin M, Gronroos TJ, et al. Extracellular superoxide dismutase is a growth regulatory medi- ator of tissue injury recovery. Mol Ther 2009;17:448-54.

20. Xiong W, Meisinger T, Knispel R, Worth JM, Baxter BT. MMP-2 regulates Erk1/2 phosphorylation and aortic dilatation in Marfan syndrome. Circ Res 2012;110:e92-e101.

21. Ha JM, Kim YW, Lee DH, Yun SJ, Kim EK, Hye Jin I, et al. Regulation of arterial blood pressure by Akt1-dependent vascular relaxation. J Mol Med 2011;89:1253-60.

22. Chung AW, Au Yeung K, Cortes SF, Sandor GG, Judge DP, Dietz HC, et al. Endothelial dysfunction and compromised eNOS/Akt signaling in the thoracic aorta during the progression of Marfan syndrome. Br J Pharmacol 2007;150:1075-83.

23. Ushio-Fukai M, Alexander RW, Akers M, Yin Q, Fujio Y, Walsh K, et al. Reactive oxygen species mediate the activation of Akt/protein kinase B by angiotensin II in vascular smooth muscle cells. J Biol Chem 1999;274:22699-704.

24. Phillippi JA, Klyachko EA, Kenny JP 4th, Eskay MA, Gorman RC, Gleason TG. Basal and oxidative stress-induced expression of metallothionein is decreased in ascending aortic aneurysms of bicuspid aortic valve patients. Circulation 2009;119:2498-506.

25. Boyd NL, Park H, Yi H, Boo YC, Sorescu GP, Sykes M, Jo H. Chronic shear induces caveolae formation and alters ERK and Akt responses in endothelial cells. Am J Physiol Heart Circ Physiol 2003;285: H1113-22.

26. Balistreri CR, Pisano C, Candore G, Maresi E, Codispoti M, Ruvolo G. Focus on the unique mechanisms involved in thoracic aortic aneurysm formation in bicuspid aortic valve versus tricuspid aortic valve patients: clinical implications of a pilot study. Eur J Cardiothorac Surg 2013;43: e180-6.

27. Jones JA, Stroud RE, Kaplan BS, Leone AM, Bavaria JE, Gorman JH 3rd, Gorman $\mathrm{RC}$, et al. Differential protein kinase $\mathrm{C}$ isoform abundance in ascending aortic aneurysms from patients with bicuspid versus tricuspid aortic valves. Circulation 2007;116:I144-9. 